\title{
Safe Fusion Compared to Established Distributed Fusion Methods
}

\author{
Jonas Nygårds, Viktor Deleskog and Gustaf Hendeby
}

Conference Publication

\section{Tweet}

N.B.: When citing this work, cite the original article.

Original Publication:

Jonas Nygårds, Viktor Deleskog and Gustaf Hendeby, Safe Fusion Compared to Established Distributed Fusion Methods, Proceedings of IEEE International Conference on Multisensor Fusion and Integration for Intelligent Systems, 2016.

Copyright: http://ieee.org

Postprint available at: Linköping University Electronic Press

http://urn.kb.se/resolve?urn=urn:nbn:se:liu:diva-131425

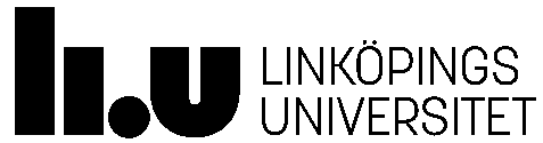




\title{
Safe Fusion Compared to Established Distributed Fusion Methods
}

\author{
Jonas Nygårds*, Viktor Deleskog*, and Gustaf Hendeby ${ }^{\dagger}$ \\ * Div. of C4ISR, Swedish Defence Research Agency (FOI), Linköping, Sweden \\ e-mail: firstname. lastnamedfoi.se \\ $\dagger$ Dept. of Electrical Engineering, Linköping University, Linköping, Sweden \\ e-mail: hendeby@isy.liu.se
}

\begin{abstract}
The safe fusion algorithm is benchmarked against three other methods in distributed target tracking scenarios. Safe fusion is a fairly unknown method similarly to, e.g., covariance intersection, that can be used to fuse potentially dependent estimates without double counting data. This makes it suitable for distributed target tracking, where dependencies are often unknown or difficult to derive. The results show that safe fusion is a very competitive alternative in five evaluated scenarios, while at the same time easy to implement and compute compared to the other evaluated methods. Hence, safe fusion is an attractive alternative in track to track fusion systems.
\end{abstract}

\section{INTRODUCTION}

Methods for track-to-track fusion (T2TF) are important in distributed tracking systems. T2TF enables tracks from multiple sources to be fused in a close to optimal way. Nowadays it is also common that sensors contains some sort of tracking functionality which limits access to the "raw" sensor output. To integrate such a sensor in a tracking network of different sensors T2TF is necessary.

In the literature, different methods for $\mathrm{T} 2 \mathrm{TF}$ have been presented and analyzed. In this paper we implement and compare four different methods, with focus on robustness and tracking accuracy and how they perform against centralized measurement fusion (CMF), which is the optimal choice if possible. The methods studied in this paper are: (i) nä̈ve information matrix fusion (naïve IMF); (ii) covariance intersection (CI) fusion [5, 11]; (iii) generalized information matrix filter (GIMF) fusion [19], and (iv) safe fusion (SF) [9]. The authors have not manged to find any publication where SF (or equivalent methods), contrary to the other three methods, have been applied to the T2TF problems. However, the ellipsoidal intersection (IE, [15, 17]) method, as mentioned below to produce the same result as SF, has been evaluated in other contexts, e.g., vehicle platooning in [16]. One of the contributions of this paper is hence to compare SF to other methods in the context of T2TF.

To get optimal T2TF performance the cross-correlations between tracks must be taken into account [18], one unavoidable source of cross-correlations is the shared process noise in tracks that describe the same target. The naïve fusion method assumes that tracks are uncorrelated which leads to overconfident error covariance matrices and overuse of data. Both CI (and variations thereof, [3, 14]) and SF assumes that there exist an unknown cross-correlation between tracks and provides a conservative solution, which is a more sound approach than naïve fusion. Exact methods as those in [18], on the other hand, calculates the cross-correlations between tracks and attains optimal performance, with the drawback that it requires a lot of information to be transferred between sensors. The ellipsoidal intersection method combines the two approaches, approximating the cross-correlation with a worst case scenario and then compensate for it. Though, derived based on different fundamental principles, it turns out that SF and EI produces identical estimates. The GIMF method also uses an information theoretic approach to handle the crosscorrelation. It is known that information is additive, hence you can subtract information to avoid double counting data.

In the real world, communication between nodes in a network is not ideal, especially in wireless network configurations with limited communication rate and possible communication delays. Such issues are a current focus in T2TF community. Here, we consider communication to be mostly synchronous, in the sense that data is current when fusing, but not necessarily at full rate. In some cases delayed measurements are also considered.

In recent research issues regarding communication rate and transmission load are highlighted to pursue CMF performance. For example the distributed version of the augmented state density (ASD) filter called DASD [13] and the recent developed distributed Kalman filter (DKF) [7]. In [6], the two previous methods are compared in terms of fusion performance, process noise sensitivity, and level of global knowledge about sensor parameters.

The main contribution of this paper is to compare the SF algorithm to other T2TF methods in a security setting, this way bringing the attention to the SF method. To evaluate the chosen T2TF methods we have picked three different datasets to accentuate the differences between the fusion methods. The first dataset is based on data used in previous T2TF evaluations [19]; the second dataset consists of recorded target trajectories [20]; and the third dataset is data from a real world field trial suitable for target tracking in surveillance applications highlighting security problems.

The paper is organized as follows. In Sec. II the general notation and description of methods are presented. How the evaluation of the methods was performed is presented in Sec. III and the results and discussion in Sec. IV. Sec. V 


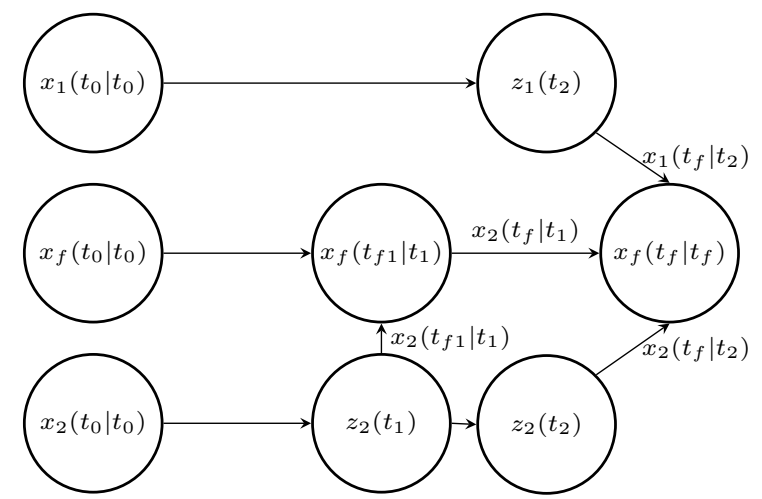

Fig. 1. Sampling sequence for asynchronous fusion of two sensors.

concludes the paper.

\section{Method Description}

It is assumed that bandwidth restrictions in combination with communication delays leads to an architecture where not every sample is communicated between the sensor locations. Consider a generic asynchronous case of decentralized fusion, as depicted in Fig. 1. We have two sensors and a fusion center that could be co-located with one of the sensors (or both if fully decentralized). At time $t_{2}$ we have new information from the first sensor $z_{1}\left(t_{2}\right)$ which is brought forward (under communication delays) to the fusion center as the prediction $x_{1}\left(t_{f} \mid t_{2}\right)$ and since we assume asynchronous reports we have also the current and predicted values for the the second sensor; i.e. information at the time of the most current sample and at the previous communication time $x_{2}\left(t_{f} \mid t_{2}\right)$ and $x_{2}\left(t_{f} \mid t_{1}\right)$, respectively. To keep the notation minimal we will in the following drop the argument for the most recent estimates from the two sensors and only keep it for the previous information for the second sensor. We assume that the estimates can be represented by their first two moments thus $\left\{\hat{x}_{1}, P_{1}\right\}$, $\left\{\hat{x}_{2}, P_{2}\right\},\left\{\hat{x}_{f}, P_{f}\right\}$ and $\left\{\hat{x}_{2}\left(t_{f} \mid t_{1}\right), P_{2}\left(t_{f} \mid t_{1}\right)\right\}$ are the notation for the variables considered in the track fusion steps. In the following sections brief descriptions of the studied methods are given, for more information the reader is referred to the provided main references of the methods.

All methods are assumed to have some sort of fusion memory, i.e. a state that keeps the predicted first two moments from the last fusion at the fusion center. For GIMF this is already included, but also for CIF and SF this memory is used to enable the use of asynchronous sensor information.

\section{A. Local and Centralized Filters}

As reference a centralized extended Kalman filter (EKF) [12] is run on all measurements from the sensors. The EKF is also used as the local filter in the sensor nodes for most scenarios. For one scenario an interacting multiple models (IMM, [4]) filter is used with a bank of two EKFs tuned with a low and a high process noise level. The model transition probability matrix in the IMM is tuned for sojourn times of
$30 \mathrm{~s}$ for the low noise and $8 \mathrm{~s}$ for the high noise case following the model in [1] giving the transition probability matrix:

$$
\Pi=\left[\begin{array}{ll}
0.9983 & 0.0017 \\
0.0062 & 0.9938
\end{array}\right]
$$

\section{B. Generalized Information Matrix Filter}

The GIMF is a generalization of the information filter for asynchronous tracklets following [19]. At the time of fusion the previous information is redacted giving the resulting equations:

$$
\begin{aligned}
P_{f}^{-1} & =P_{1}^{-1}+\left[P_{2}^{-1}-P_{2}\left(t_{f} \mid t_{1}\right)^{-1}\right] \\
P_{f}^{-1} \hat{\boldsymbol{x}}_{f} & =P_{1}^{-1} \hat{\boldsymbol{x}}_{1}+\left[P_{2}^{-1} \hat{\boldsymbol{x}}_{2}-P_{2}\left(t_{f} \mid t_{1}\right)^{-1} \hat{\boldsymbol{x}}_{2}\left(t_{f} \mid t_{1}\right)\right] .
\end{aligned}
$$

The decorrelation by removing the previous information can also be performed at the local estimates as in the channel filter [8].

\section{Covariance Intersection}

The CI fusion rule [11] was explicitly developed to handle the problem of fusion of two (Gaussian) estimates with unknown correlations. The fusion equations are

$$
\begin{aligned}
P_{f}^{-1} & =\omega P_{1}^{-1}+(1-\omega) P_{2}^{-1} \\
P_{f}^{-1} \hat{\boldsymbol{x}}_{f} & =\omega P_{1}^{-1} \hat{\boldsymbol{x}}_{1}+(1-\omega) P_{2}^{-1} \hat{\boldsymbol{x}}_{2},
\end{aligned}
$$

where

$$
\omega=\underset{\omega}{\arg \min } \operatorname{det}\left(P_{f}\right) .
$$

The choice to use the determinant in the criteria can be seen as minimizing the Shannon information [10].

\section{Nä̈ve Independence Assumption}

For comparison the result of applying naïve fusion, i.e., fusion of the track reports ignoring the correlation introduced by the common process noise in the target trajectory, are also presented. The corresponding fusion equations for the naïve information matrix filter (naïve IMF) are:

$$
\begin{aligned}
P_{f}^{-1} & =P_{1}^{-1}+P_{2}^{-1} \\
P_{f}^{-1} \hat{\boldsymbol{x}}_{f} & =P_{1}^{-1} \hat{\boldsymbol{x}}_{1}+P_{2}^{-1} \hat{\boldsymbol{x}}_{2}
\end{aligned}
$$

This filter is thus the information form of the Kalman filter [12] under the (for track to track fusion naïve) assumption of uncorrelated errors between the local tracks.

\section{E. Safe Fusion}

Similar to the covariance intersection method, safe fusion (SF, [9]) avoids double counting information from two possibly dependent estimates by decoupling the components in the estimates and using the most informative one from each estimate. This is achieved by repeatedly applying singular value decomposition (SVD). The largest ellipsoid method [3] utilizes an eigen vector basis factorization to obtain the same covariance matrix as SF, but differs in how the mean of the estimate is computed. Contrary to CI, SF has not been shown to provide consistent estimates. 


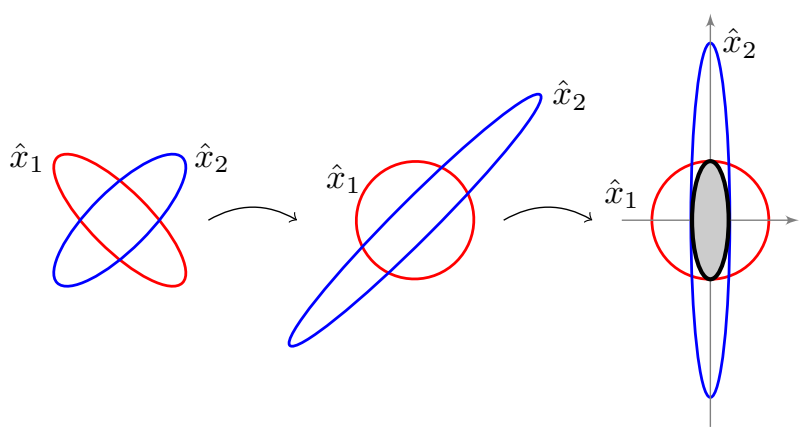

Fig. 2. Illustration of the important steps of safe fusion between the two possibly correlated estimates $\hat{x}_{1}$ and $\hat{x}_{2}$.

This is illustrated in Fig. 2. To the very right in the figure, the two estimates, $\hat{\boldsymbol{x}}_{1}$ and $\hat{\boldsymbol{x}}_{2}$, to be fused are illustrated by their covariance ellipses. In step 1 of the algorithm, a linear transformation is applied (obtained from an SVD) to transform the covariance matrix of $\hat{\boldsymbol{x}}_{1}$ into a unit matrix (the middle of the illustration). The components in $\hat{\boldsymbol{x}}_{1}$ are now independent. In step 2, a rotation is applied (obtained by an SVD) to make the components in $\hat{\boldsymbol{x}}_{2}$ independent (the right part of the illustration). Note that the components in $\hat{\boldsymbol{x}}_{1}$ remain independent under the rotation as they are equally uncertain in all directions. Next the most informative estimate is used in each direction, resulting in the grayed ellipsoid to the right. It is allowed to treat the components independently as the different directions have been decoupled by the two transformations. Double counting information is hence avoided by using only information from one of the two estimates to be fused in each direction. Finally, the fused estimate is obtained by simply applying the inverse of the two transformations (not illustrated).

For completeness, the algorithm is provided in Algorithm 1, explicitly stating how to derive the necessary transformations. It should be noted that SF can be implemented using standard linear algebra functions, without the requirement of the optimization step found in CI. Hence, as SF also does not need to store or compute correlations it can be efficiently implemented with predictable execution time. The interested reader is referred to [9] for details and a motivation.

\section{Method Evaluation}

The methods will be evaluated through three different datasets. The first two datasets are chosen from literature to allow for comparisons. The first set is inspired by [19] to provide direct comparisons for the GIMF. The second set is an adaption of one of the air scenarios in [20] (for brevity called Blair in the figures) used as a ground person tracking scenario. Finally results from field trials of a security scenario are presented. For the second dataset permutations in parametrizations of the process models or the introduction of IMM models gives two additional scenarios for a total of 5 scenarios described below.
Algorithm 1 Safe Fusion [9]

Given two possibly correlated estimates of $x, \hat{\boldsymbol{x}}_{1}$ and $\hat{\boldsymbol{x}}_{2}$ such that $P_{1}=\operatorname{cov}\left(\hat{\boldsymbol{x}}_{1}\right)$, and $P_{2}=\operatorname{cov}\left(\hat{\boldsymbol{x}}_{2}\right)$ :

1) Compute $U_{1}$ and $D_{1}$, using an SVD of the positive definite matrix $P_{1}$, such that

$$
P_{1}=U_{1} D_{1} U_{1}^{T}
$$

2) Similarly, derive $U_{2}$ and $D_{2}$ using an SVD, such that

$$
D_{1}^{-1 / 2} U_{1}^{T} P_{2} U_{1} D_{1}^{-1 / 2}=U_{2} D_{2} U_{2}^{T} .
$$

3) Let

$$
\begin{aligned}
T & =U_{2}^{T} D_{1}^{-1 / 2} U_{1}^{T} & \\
\hat{\overline{\boldsymbol{x}}}_{1} & =T \hat{\boldsymbol{x}}_{1} & \hat{\overline{\boldsymbol{x}}}_{2}=T \hat{\boldsymbol{x}}_{2},
\end{aligned}
$$

where by construction $\operatorname{cov}\left(\hat{\overline{\boldsymbol{x}}}_{1}\right)=I$ and $\operatorname{cov}\left(\hat{\overline{\boldsymbol{x}}}_{2}\right)=D_{2}$.

4) Select the most informative source for each component $i=1,2, \ldots, \operatorname{dim}(x)$, let

$$
\begin{array}{llll}
{[\hat{\overline{\boldsymbol{x}}}]_{i}=\left[\hat{\overline{\boldsymbol{x}}}_{1}\right]_{i},} & {[D]_{i i}=1} & \text { if } & {\left[D_{2}\right]_{i i} \geq 1,} \\
{[\hat{\overline{\boldsymbol{x}}}]_{i}=\left[\hat{\overline{\boldsymbol{x}}}_{2}\right]_{i},} & {[D]_{i i}=D_{2}^{i i}} & \text { if } & {\left[D_{2}\right]_{i i}<1 .}
\end{array}
$$

5) The final estimate given by

$$
\begin{aligned}
& \hat{\boldsymbol{x}}_{f}=T^{-1} \hat{\overline{\boldsymbol{x}}} \\
& P_{f}=T^{-1} D^{-1} T^{-T} .
\end{aligned}
$$

\section{A. Scenario 1}

The first setup is basically the same as Scenario 3 in [19], but with the same sample time for both sensors and without initial delay. Thus the scenario consist of two sensors one at origin and one at $(5000 \mathrm{~m}, 0 \mathrm{~m})$. They sample the position with $2 \mathrm{~s}$ interval ([19] uses 2 and $2.5 \mathrm{~s}$ ). The range-bearing uncertainty is modeled as white noise with standard deviation $\sigma_{r}=10 \mathrm{~m}$ in range and $\sigma_{\theta}=1^{\circ}$ in bearing. The fusion is only performed with a rate of $8 \mathrm{~s}$ and a delay of $8 \mathrm{~s}$. The motion model used is a continuous white noise acceleration model (CWNA) with the process noise $\sigma_{w}=0.1 \mathrm{~ms}^{-3 / 2}$.

In the first scenario the process model used for the motion is the same as the one used in the filter, hence the centralized Kalman filter is optimal. However, for a typical security scenario the human motion model is more like the aircraft flight of [20].

\section{B. Scenario 2}

For the second scenario we modify flight Scenario 6 of [20] to a ground scenario by scaling both positions and velocities with $1 / 1000$ giving a small velocity but reasonable motions for a ground scenario. (See Fig. 3.) The sensors are placed at $(0 \mathrm{~m}, 5 \mathrm{~m})$ and $(0 \mathrm{~m}, 45 \mathrm{~m})$, respectively, and sampled at $5 \mathrm{~Hz}$. It runs without any delay but with fusion in a subsampled rate of $2.5 \mathrm{~s}$. The motion model is still a CWNA with the process noise $\sigma_{w}=0.1 \mathrm{~ms}^{-3 / 2}$. 


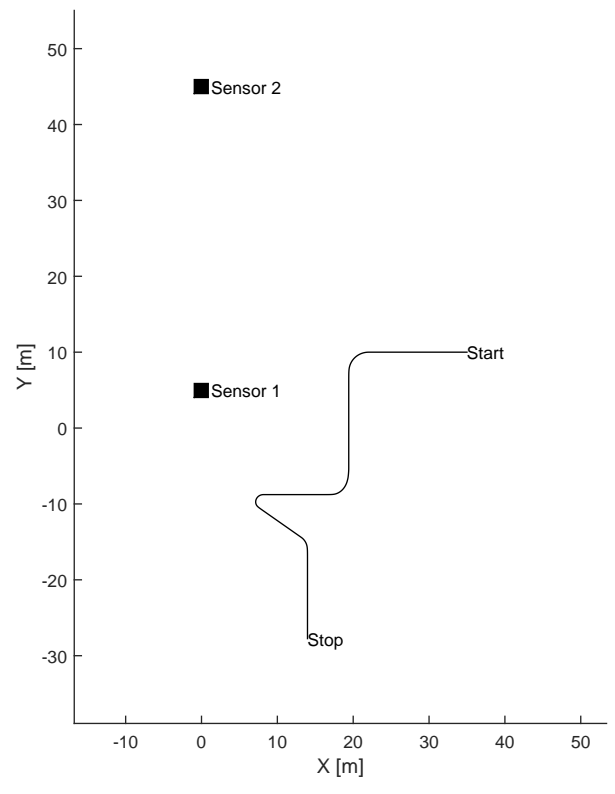

Fig. 3. Trajectory of Scenario 6 in [20] adopted to a ground scenario.

\section{Scenario 3}

The third scenario is identical to Scenario 2, except for an additional delay of $2.5 \mathrm{~s}$.

\section{Scenario 4}

In Scenario 4 we return to Scenario 2 but introduces the IMM filter for the local sensors where the high/low (H/L) process models are chosen as $\sigma_{w H}=4 \times 0.1 \mathrm{~ms}^{-3 / 2}$ and $\sigma_{w L}=0.1 / 4 \mathrm{~ms}^{-3 / 2}$, respectively.

\section{E. Scenario 5}

The final scenario illustrates a real world example of tracking a person in surveillance cameras. There are two cameras pointing at the same area from different angles where one person walks through as illustrated in Fig. 4. The person is assumed to move according to a constant velocity model with process noise $\sigma_{w}=0.2 \mathrm{~ms}^{-3 / 2}$. The fusion runs at a rate of $0.5 \mathrm{~s}$.

\section{F. Evaluation}

The results are evaluated against ground truth when available. Monte Carlo simulations are performed with 100 samples for Scenario 1 and 40 samples for Scenarios 2-4. To allow easy comparison with [19] the solutions of Scenario 1 are tested for consistency by normalized state estimation error squared (NEES, [2])

$$
\operatorname{NEES}(t)=\left(x(t)-\hat{\boldsymbol{x}}_{f}(t \mid t)\right)^{T} P_{f}^{-1}(t \mid t)\left(x(t)-\hat{\boldsymbol{x}}_{f}(t \mid t)\right),
$$

evaluated as a mean over Monte Carlo evaluations.

The performance is evaluated as root mean square error (RMSE) averaged over Monte Carlo evaluations in Scenarios 1-4. In Scenario 5 no ground truth is available so the results will be evaluated as the root mean square deviation (RMSD)

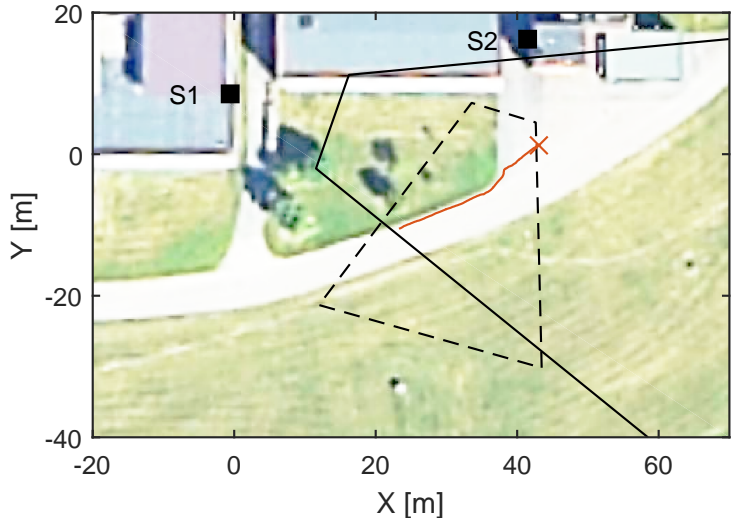

Fig. 4. Map overview of Scenario 5 where the optimal track is marked as red. The two sensors, S1 and S2, are illustrated as black boxes. The field of view of each sensor is illustrated as lines for S1 and dashed lines for S2. The area considered for evaluation is the common area seen by both sensors. The target moves from left to right.

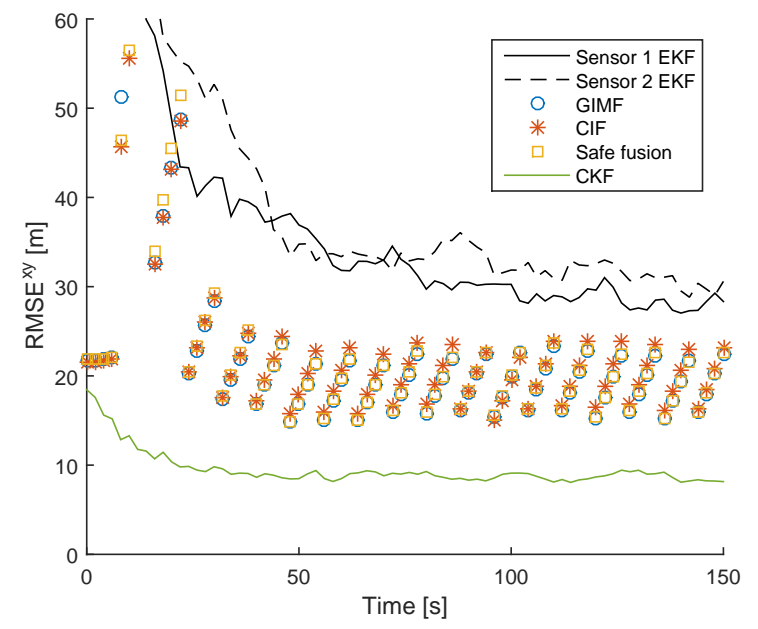

Fig. 5. 100 Monte Carlo simulations on a continuous white noise acceleration scenario similar to [19].

from the optimal track, i.e. the CKF. The optimal track and each sensor track is generated using a multi-sensor-multi-target tracker which associates visual detections from each sensor to tracks in a world coordinate frame. In the project both performance measures were studied for all scenarios but for brevity only the most interesting plots are reproduced here.

\section{RESULTS AND DisCUSSION}

Scenario 1 was chosen to relate the obtained results to the results in [19]. The result in Fig. 5 compare favorably with the results in [19], but note that the initialization here was not as advanced as in the initial reference, causing larger errors for the first two updates in our implementation. The SF filter gives comparable results with the GIMF while the CIF performance varies along the trajectory. In Fig. 6 the consistency of the fusion is tested by the NEES. Apart from the inconsistent initialization, both the GIMF and SF filter 


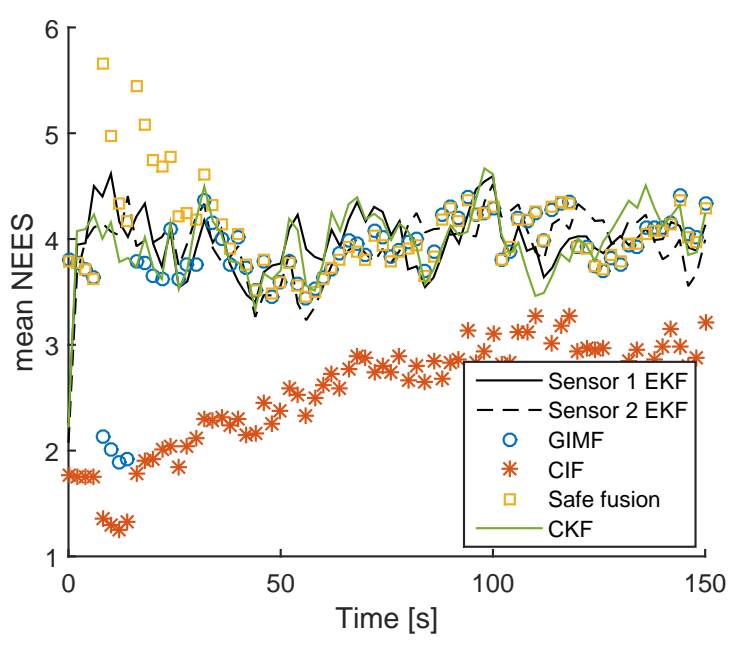

Fig. 6. Mean NEES of 100 Monte Carlo simulations on a continuous white noise acceleration scenario similar to [19].

perform consistently while as expected the CI show an overly conservative covariance.

The grouping of estimates four by four is due to the reduced rate of information used for the fusion. New information only arrives every $8 \mathrm{~s}$, i.e., every fourth sample.

For the second dataset in Scenario 2-4 with the trajectory illustrated in Fig. 3 the true motion model is nonlinear and thus the baseline CKF is no longer the optimal filter but can only aspire to be the best linear filter. In this case the local filters will not necessarily provide consistent estimates at all times either. In Fig. 7 the performance for Scenario 2, without delay is illustrated. Here the SF performs better than the CKF that actually cannot be optimal for this scenario. Since the path is based on basically linear motion with maneuvers the use of a CWNA has to be a compromise between good tracking in the corners or along the straight lines. It is interesting to note that for the more linear parts of the paths the SF is actually better than the CKF. However in the corners when the local filters lag the SF has worse performance but still at the same level as the GIMF. When delays are introduced as in Fig. 8 in Scenario 3 the SF can no longer beat the CKF but actually reach the level of CKF on the straight lines. In the corners the decentralized filters give worse performance than the individual local filters this is probably due to the predictions of the local states being inconsistent due to small process variance in the linear model during corners.

The naïve IMF actually has performance worse than the local filters in the corners.

The results suggest that an IMM would actually perform better, hence in Fig. 9 IMM filters have been applied both on the local and central level. On the central level the IMM does not improve the situation as much as expected on the straight lines, probably due to relatively uncertain sensors. For the local filters, especially of sensor 1, an improvement can be seen which also translates to an improvement for the fused filters. Now at the update times the filters are better than the local estimates. Again the SF filter is better than the central IMM filter on the straight line parts. Using local IMM filters complicated the picture and no straightforward way to implement exact methods [18] was seen, but even the GIMF poses problems in which model to use for the predictions. In the results presented, the model with the large process noise was used to predict the previous information forward. Trials using smaller variance made the filter unstable during shifts in the modes between large and small process noise. Here the SF and CI filters were the only ones that were straight-forward to implement and since the SF filter is more consistent than the CI with a less conservative covariance it would be the better choice.

In Fig. 10 the performance for Scenario 5 is illustrated with a fusion rate set to $2 \mathrm{~Hz}$. Here the GIMF filter performs best, especially at the fusion points, with the smallest deviation from CKF. This was the expected result since GIMF is optimal at full rate. Here, both SF, CIF, and naïve IMF show almost equal performance. The naïve assumption shows almost no degradation in performance as in previous scenarios. This could be an effect of the simple target movements in the scenario. When the estimated error covariance is inspected, naïve IMF instead turns out to be overconfident contrary to the other methods, which is due to the information double counting. The scenario does not cover advanced target trajectories as in the previous scenarios, but it shows once again that SF is an applicable method for T2TF.

\section{CONCLUSION}

In this paper a less known alternative to the covariance intersection (CI) method for fusion of correlated estimates, safe fusion (SF), was evaluated using simulated and a real world experimental data. It provides a less conservative covariance than the $\mathrm{CI}$ method, hence it provides estimates with better consistency. In the scenarios of interest, motivated by camera surveillance scenarios, the SF performed well, on a level comparable to established methods such as generalized information matrix fusion (GIMF). The algorithm works well in conjunction with local interacting multiple models (IMM) filters on level with the GIMF and where the exact methods [18] are intractable. SF can be implemented using standard linear algebra methods and has predictable computation time, making it an attractive alternative to the other described methods. When local IMM filters are used, careful considerations need to be taken in the choice of process model for the prediction used for the GIMF making the SF the more robust alternative.

\section{ACKNOWLEDGMENTS}

This paper was supported by research projects at the Swedish Defence Research Agency (FOI) funded by the Swedish Armed Forces. G. Hendeby was supported by The Swedish Research Council through their grant Scalable Kalman Filters. The authors would also like to thank the anonymous reviewers for insightful comments and for pointing 


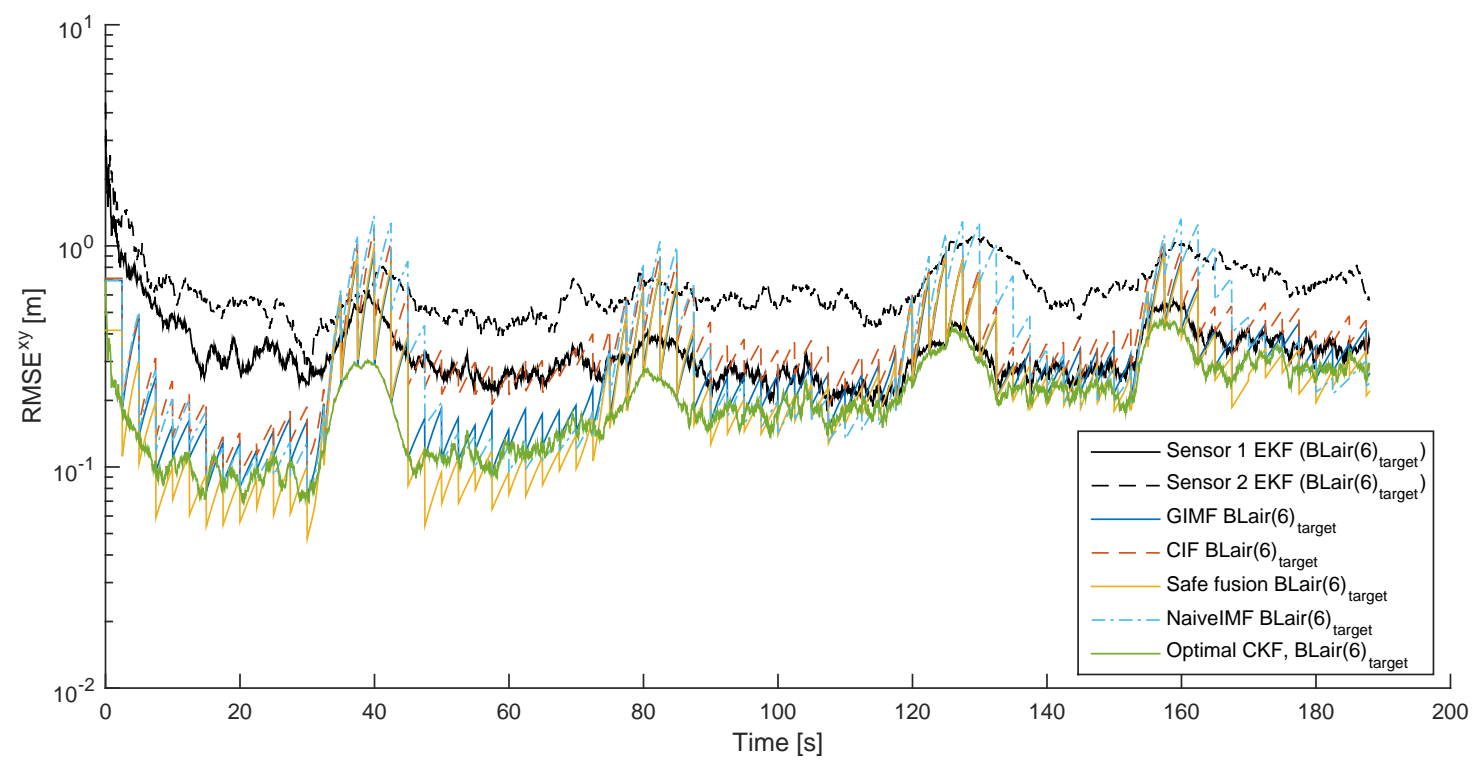

Fig. 7. Root mean square error for the trajectory of Fig. 3

to the ellipsoid intersection method and its relations to safe fusion.

\section{REFERENCES}

[1] Y. Bar-Shalom and H. Chen. Covariance reconstruction for track fusion with legacy track sources. Journal of Advances in Information Fusion, 2008.

[2] Y. Bar-Shalom, X. R. Li, and T. Kirubarajan. Estimation with applications to tracking and navigation: theory algorithms and software. John Wiley \& Sons, 2004.

[3] A. R. Benaskeur. Consistent fusion of correlated data sources. In 28th Annual Conference of the Industrial Electronics Society, volume 4, pages 2652-2656, Nov. 2002.

[4] S. Blackman and R. Popoli. Modern tracking systems. Artech House, $1,1999$.

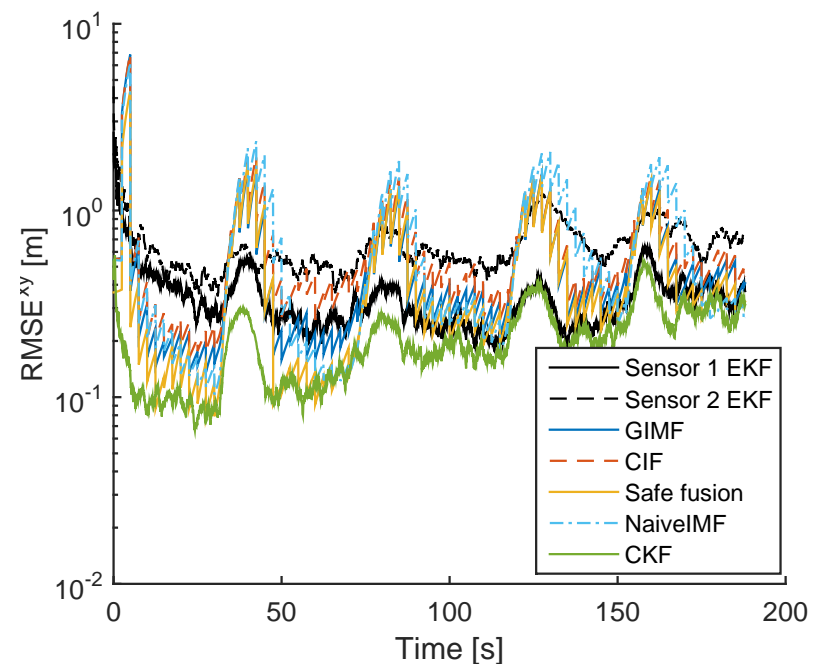

Fig. 8. Mean square error for delayed local EKF filters for the trajectory of Fig. 3. The legend is the same as in Fig. 7
[5] L. Chen, P. O. Arambel, and R. K. Mehra. Fusion under unknown correlation - covariance intersection as a special case. In Proceedings of 7th IEEE International Conference on Information Fusion, pages 905-912, Annapolis, MD, USA, July 2002.

[6] C.-Y. Chong, W. Koch, and F. Govaers. Comparsion of tracklet fusion and distributed kalman filter for track fusion. In Proceedings of 17th IEEE International Conference on Information Fusion, 2014.

[7] F. Govaers and W. Koch. Distributed kalman filter fusion at arbitrary instants of time. In Proceedings of 13th IEEE International Conference on Information Fusion, 2010

[8] S. Grime and H. F. Durrant-Whyte. Data fusion in decentralized sensor networks. Control engineering practice, 2(5):849-863, 1994.

[9] F. Gustafsson. Statistical Sensorfusion. Studentlitteratur, 2010.

[10] M. B. Hurley. An information theoretic justification for covariance intersection and its generalization. In Proceedings of 7th IEEE International Conference on Information Fusion, volume 1, Annapolis, MD, USA, 
July 2002.

[11] S. J. Julier and J. K. Uhlmann. A non-divergent estimation algorithm in the presence of unknown correlations. In Proceedings of American Control Conference, pages 2369-2373, Albuquerque, NM, USA, June 1997.

[12] T. Kailath, A. H. Sayed, and B. Hassibi. Linear Estimation. PrenticeHall, Inc, 2000. ISBN 0-13-022464-2.

[13] W. Koch and F. Govaers. On decorrelated track-to-track fusion based on accumulated state densities. In Proceedings of 17th IEEE International Conference on Information Fusion, 2014.

[14] W. Niehsen. Information fusion based on fast covariance intersection filtering. In Proceedings of 7th IEEE International Conference on Information Fusion, volume 2, pages 901-904, Annapolis, MD, USA, July 2002.

[15] B. Noack, J. Sijs, M. Reinhardt, and U. D. Hanebeck. Treatment of dependent information in multisensor Kalman filtering and data fusion. In H. Fourtati, editor, Multisensor Data Fusion: From Algorithms and
Architectural Design to Applications, page 169-192. CRC Press, 2015.

[16] J. Sijs and M. Lazar. Emperical case-study of state fusion via ellipsoidal intersection. In Proceedings of 14th IEEE International Conference on Information Fusion, Chicago, IL, July 2011.

[17] J. Sijs and M. Lazar. State fusion with unknown correlation: Ellipsoidal intersection. Automatica, 48:1847-1878, Aug. 2012.

[18] X. Tian and Y. Bar-Shalom. Exact algorithms for four track-to-track fusion configurations: All you wanted to know but were afraid to ask In Proceedings of 12th IEEE International Conference on Information Fusion, pages 537-544, 2009.

[19] X. Tian and Y. Bar-Shalom. On algorithms for asynchronous track-totrack fusion. In Proceedings of 13th IEEE International Conference on Information Fusion, pages 1-8, 2010.

[20] G. Watson and W. Blair. Benchmark problem for radar resource allocation and tracking maneuvering targets in the presence of ECM. Technical report, Technical Report NSWCDD/TR-96/10, 1996.

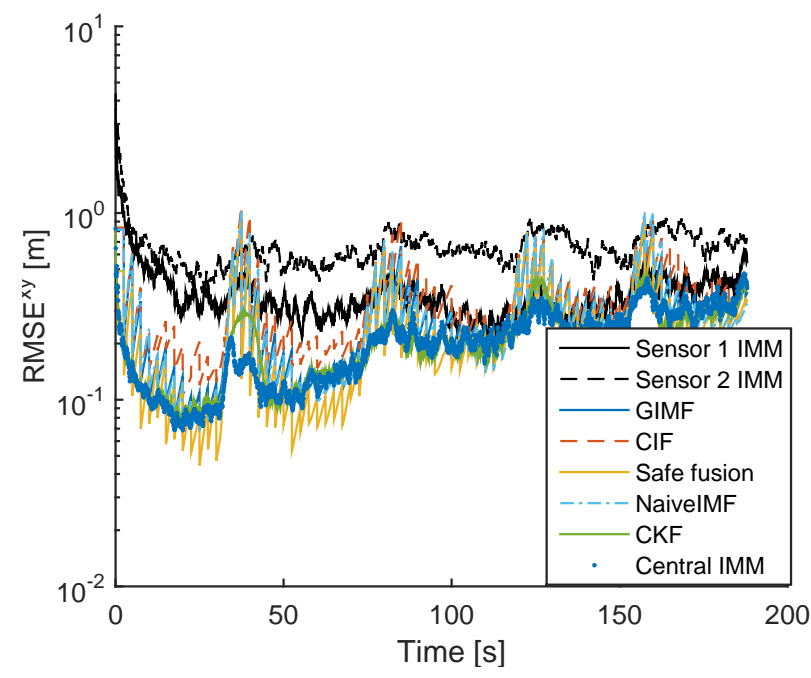

Fig. 9. The results of applying IMM filters on the trajectory of Fig. 3

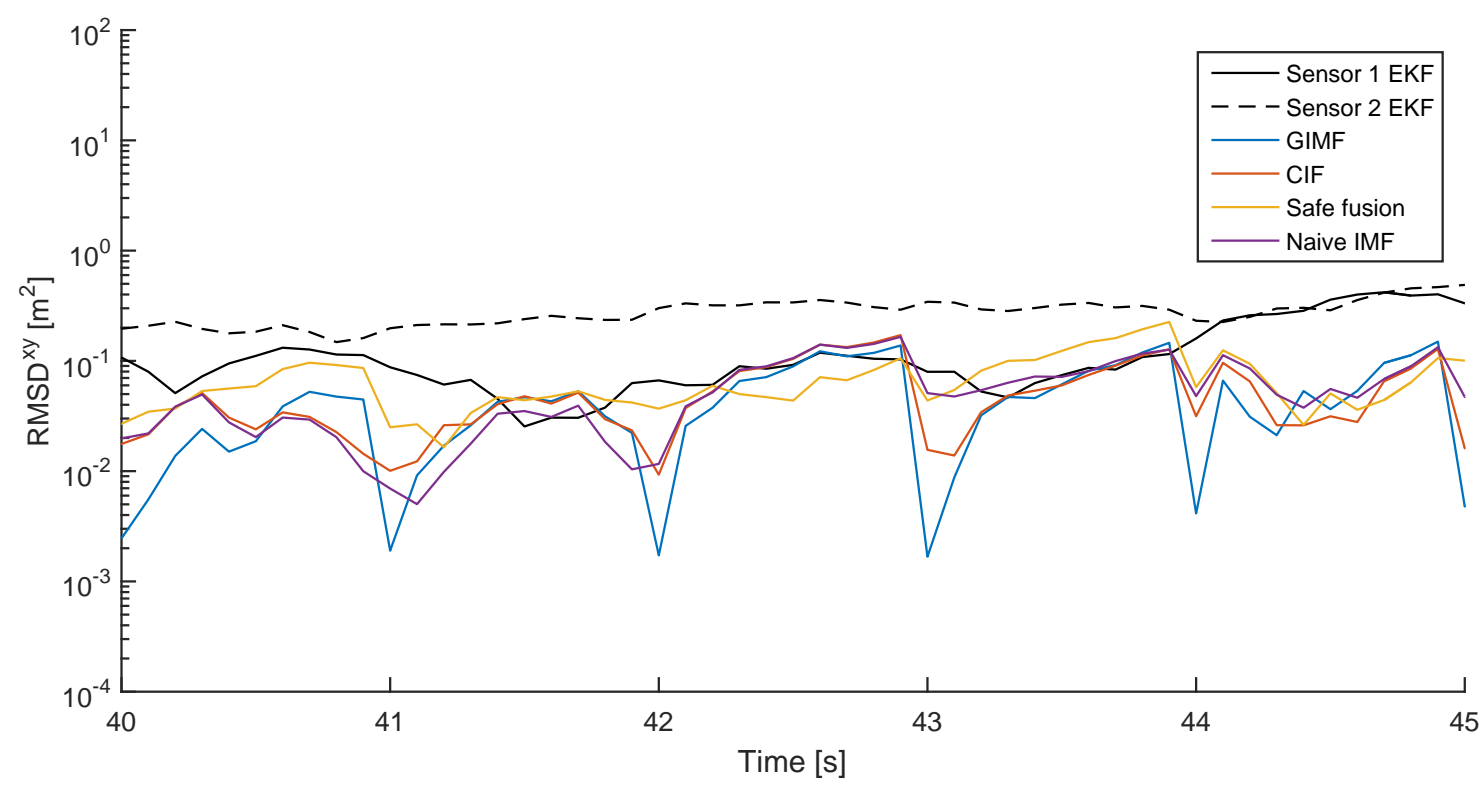

Fig. 10. RMSD from the optimal generated CKF track for the trajectory in Fig. 4. 